\title{
Digitized and Visualized Knowledge Structures in Recommending Associated Web Links
}

\author{
Athitaya Nitchot \\ Prince of Songkla International College, Prince of Songkla University \\ 12th Floor, Learning Resource Center (LRC), Square Building \\ 15 Kanjanavanich Road, Hat Yai, Songkla 90110 Thailand \\ athitaya.nitchotegmail.com
}

\begin{abstract}
E-learning and web-based learning are intended to support learners. It is still difficult, however, for learners to identify and choose study materials that match their current and desired abilities. Some e-learning systems do not provide the information needed to assist learners and avoid these difficulties. This research proposes pedagogically-informed knowledge structures, methods to construct them and design the associated application, a tool for recommending appropriate materials. Experimental studies are conducted to validate the design of the knowledge structures and the effectiveness of the tools. Learners will be expected to gain significantly higher levels of achievement by using the knowledge structures and associated tools proposed in this research.
\end{abstract}

Keywords: knowledge structure; structure visualization; self-study; digitized structure

\section{Introduction}

Knowledge Representation refers to the study of representing knowledge formally and explicitly, so supporting unambiguous knowledge sharing. In the learning and teaching environment, knowledge representation has been adopted in many ways such as: representing a content structure of learning documents [1] supporting learners in their own knowledge acquisition [2] and enabling suggestions to learners [3]. Knowledge relationships can be represented in many forms: maps, trees, networks, and graphs. They may be called concept maps, knowledge maps, and knowledge structures.

There are several methods of building knowledge structures $[4,5]$ and an interesting question is how such structures can pedagogically support learning and teaching activities. In the early stage of this research, a literature review found some methods of knowledge structure/concept map building and use in educational technology. In this research, the preliminary results were obtained. Thai school teachers were surveyed and interviewed (as discussed in Section VI, Experimental Design and Results), and the preliminary data suggested that even though knowledge structures have been recommended for many years, school teachers did not report using them in their teaching. "Mind maps" were used to support learners' conceptualization and understanding of the course content. A mind map is a rather rough note of content information, however, where the relationships among the content items are usually vague and imprecise [6]. The teachers felt that mind maps were useful and that they 
could benefit education, helping learners think about and understand the relationships in the information shown.

In normal classroom environments, the learners' prior knowledge is always varied. Some learners may not be aware of their missing knowledge or of the links and relationships between knowledge. This could affect their learning and their ability to achieve their desired learning. Saiyasombut \& Siam Voices [7] reported that Thai learners are not being well equipped with the type of knowledge and skills that will enable them to do well in the future in which many key aspects of life will require a more demanding level of literacy. These problems can be found in any classes with any subjects. Thai classrooms tend to provide similar learning environments and all learners are assumed to have the same level of knowledge [8]. Thai learners are taught to 'remember' rather than 'understand' lessons. Consequently, they have inadequate 'thinking process' and 'solving problem' skills which are important in order to learn Mathematics effectively [9].

'Resources' are also an important factor. There is still a lack of good resources for learning. E-learning is one type of resource for overcoming this problem, comprising electronicallysupported resources such as online courses, online websites, lesson videos, instructional TV, and so on. Such resources allow learners to spend more time studying and practicing/solving lesson problems on their own at times and in places which may suit them better. There are many online study materials available which learners can access at any time and any place. Learners can retrieve supplementary online resources and familiarize themselves with missing knowledge before their course starts. It is difficult, however, for learners to find and access materials which match their intended learning outcomes or abilities. Learners may not be able to identify the relationships among knowledge, or obtain the learning materials which match their abilities. This research suggests the methods for constructing knowledge structure and an application of knowledge structures to support learning and teaching. This is a tool for representing knowledge structures and suggesting learning paths and learning resources to support a learner in achieving a desired learning outcome.

\section{Knowledge Representation and Structures}

Knowledge Representation (KR) can be defined in different ways. Sowa [10] defined KR as "a multidisciplinary subject that applies theories from three fields: logic, ontology, and computation.". There are several ways of representing knowledge. A way of building a knowledge structure was introduced by Novak [11]. The NOVAK tool for building a knowledge structure is an effective tool but it is complicated and time-consuming for knowledge acquisition in an educational setting [12].

Lee, Lee, and Leu [12] proposed a better way of defining concepts and the mapping of concepts to support a learning focus. The concepts in the structure are structured into learning sequences using epistemological ordering. A series or combination of such epistemologically ordered concepts is called a 'topological graph'. Nitchot, Gilbert, and Wills [13] proposed a method of building a competence structure. The subject matter content is analyzed and each item of subject matter content is tagged with a capability and a context in order to yield a structure of competence.

Several types of knowledge structure have been proposed in the literature. A knowledge structure was developed by Kickmeier-Rust, Albert, and Steiner [14]. A node represents a knowledge state (or a task), which is the set of the available knowledge of a person at a 
particular point in time, for example, "VWX". The structure represents the set of all possible knowledge states of a learner. The prerequisite relationships are defined within this set of knowledge states, for example, a prerequisite to "VWX" is "VX". Each item of knowledge in a state represents a problem or subject matter which a learner is required to solve or understand, for example, the learner is required to achieve the node $\mathrm{V}$ before proceeding to the node VW and VX. Another example is the node "VWX" represents the learner's ability to solve the problems or understand the subject matter "V", "W", and "X". Considering each state, the front subject matter is the prerequisite of the adjacent latter subject matter.

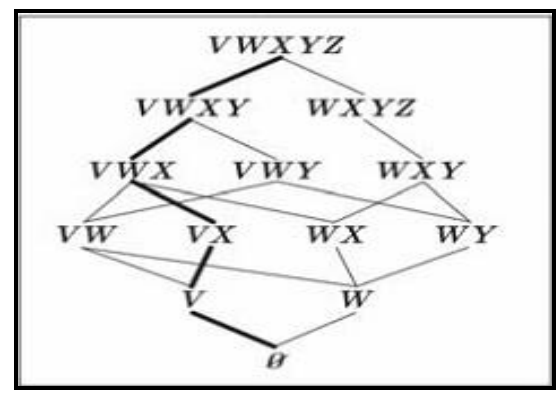

Fig. 1. Knowledge Structure Established by the Prerequisite Function [14]

Another representation of knowledge was introduced by Albert, Hockemeyer, Mayer, and Steiner [15]. This structure is illustrated in Fig1. Its design is considered in terms of structures of defined concepts and actions. According to the cognitive structural approach, skills are represented by declarative (concepts) and procedural (actions) elements. For example, the skill 'apply the Pythagorean Theorem' consists of the concept 'Pythagorean Theorem' and the action 'apply'. The concept indicates the target to which the action refers. Actions are hierarchically structured behaviours. Bloom's taxonomy [16] can be adopted for representing the actions in the educational domain. Each node of the knowledge structure contains a set of concepts and corresponding actions, and has a prerequisite relation with some other nodes. A prerequisite relation is a type of parent-child relationship where the achievement of the 'child' node is required before the 'parent' node can be achieved.

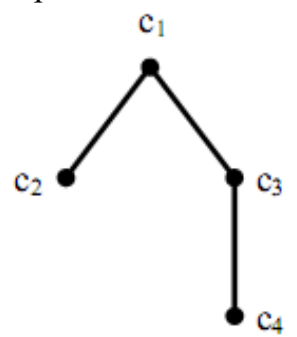

(a)

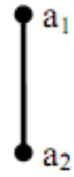

(b)

Fig. 2. Structure Defined (a) Set of Concepts and (b) Set of Actions [15]

A number of research studies have concluded that knowledge structures improve e-learning performance $[2,17]$, evaluate learners' performance and diagnose misunderstandings [18], are easy to understand [2], make information more explicit [19], and assist navigation, search, and knowledge management in web-based learning environments [20]. 


\section{Application of Knowledge Structure in Education}

A number of researchers propose the use and application of knowledge structures as an educational support system. CoMPASS software (Concept Mapped Project-Based Activity Scaffolding System) contains a hypertext system and curriculum modules based on a pedagogical framework [2]. This system supports the idea that a concept map is a way of mining knowledge from knowledge elicitations by learners. It is claimed to help learners in their thinking processes, is easy to understand, and helps learners in better understanding the courses or lessons. A similar study [21] suggests that the use of concept mapping is often linked to the constructivist view of learning, because a concept map makes a good starting point for constructivist-based teaching. Yang and Sun [1] used data mining techniques to determine the similarity of Web pages and built knowledge structures from these similarities. Similar work [22] used and integrated knowledge structures with online courses, allowing students to visualize their learning process, and be grouped with other students who shared the same learning interests. Carvalho et al. [20] developed two algorithms, SAgent and WAgent, for filtering and ranking results obtained by search engines. Their proposed algorithms are capable of identifying pages that the subjects considered relevant to their context on a map. A particular part of the map can be searched by a mobile agent that analyzes the content of the map and moves through a set of meta-search servers to carry out the search filtering and ranking. This is to provide more relevant search results.

\section{Method for Constructing Knowledge Structures}

Information on subject matter and learning outcomes has been obtained from school teachers, and a task analysis was undertaken to give a diagrammatic representation of the subject matter [23]. Knowledge structures were built by following four steps as described by Nitchot, Gilbert, and Wills [24]:

Step 1: Choose knowledge domain

To construct a competence structure, we need to consider the intended learning outcomes of the knowledge domain. For example, an intended learning outcome 'to define a graph theory' within the knowledge domain 'mathematics under high school level'

Step 2: Undertake task analysis of subject matter

Next, we summarise all the intended learning outcomes into a list of subject matter items. The first step is to consider the structure of the subject matter content in an e-learning system. This is undertaken by focusing on the broad understanding of the knowledge and cognitive skills of students, in order to achieve the goal. This is called in short 'subject matter content' and is normally categorized into four fields based on Merrill's analysis CDT [25] as fact, concept, procedure and principle. Task analysis provides the relationships and structures of subject matter. At this stage, each type of subject matter is considered as a diagrammatic approach [23]. Each category of subject matter has different notation representing its task analysis.

Task analysis of all subject matters is then levelled and the relationships are assigned. The number of levels depends on the knowledge domain.

Step 4: Structure the subject matter

An initial set of knowledge structures have been created. During step 4, levels and relationships of designed task analysis are obtained. This information is considered. All 
subject matters are represented as one node, and structured. The same levels of task analysis of subject matters are in the same levels within the structure. The relationship between subject matter nodes is parent-child. An arrow points to a child node. The structure of subject matter is obtained in step 4. In this structure, one node represents only subject matter. In order to develop a structure of subject matter to a competence structure, each node of subject matter requires tagging with a corresponding capability and a context. Fig 3 shows a sample of an initial knowledge structure of a mathematical subject (at high school level).

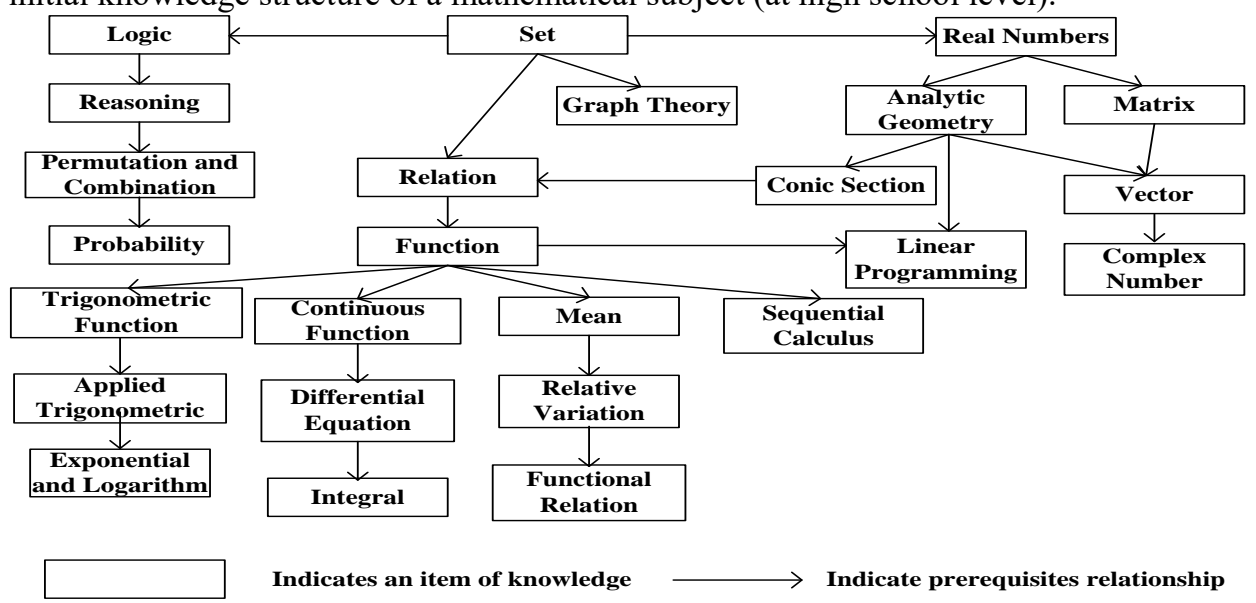

Fig. 3. Structure Defined (a) Set of Concepts and (b) Set of Actions [15]

\section{Tools for Suggesting Learning Resources' Links}

A tool for suggesting learning resources' links based on knowledge structures has been implemented as a prototype. Of the features identified, a recommender system and a learning path service have been implemented. The current tool incorporates the designed knowledge structures and their associated learning resources (mainly html links). The graph visualization libraries (such as Graphviz [26] and Microsoft Automatic Graph Layout [27]) display the graph nodes and edges from the knowledge database. The Google API is used to gather links from the web. Currently, two features as recommender system and learning path service are under investigation. (rounded rectangles with strong line).

Fig. 5 and Fig 6. show screenshots of the prototype tool in use. In Fig. 5, the chosen knowledge structure is shown and the corresponding links of associated nodes are suggested. The links are obtained from a Google search using the Google API, where the search keywords are extracted from the knowledge keywords in a node and a Google default search is considered within this research. A list of websites (e.g., YouTube, Wikipedia) is given to the users for filtering the search results. In Fig 6., the nodes are suggested based on the current and previously visited nodes. 


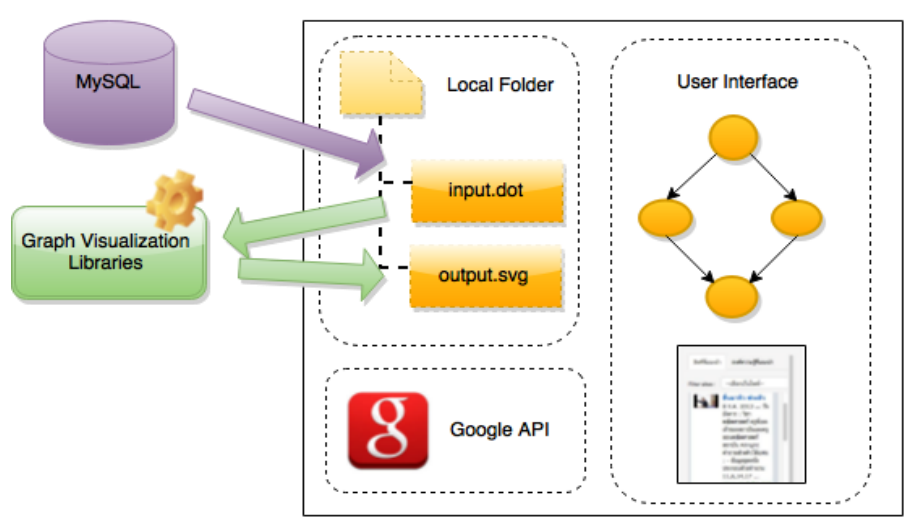

\section{Features}

Knowledge Structure

Authoring Tool

Recommender System

(a)

Learning Path Service

Sharable Knowledge Structure

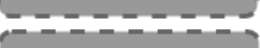

Missing Prerequisite Service

Fig. 4. Tool Infrastructure in this Proposal

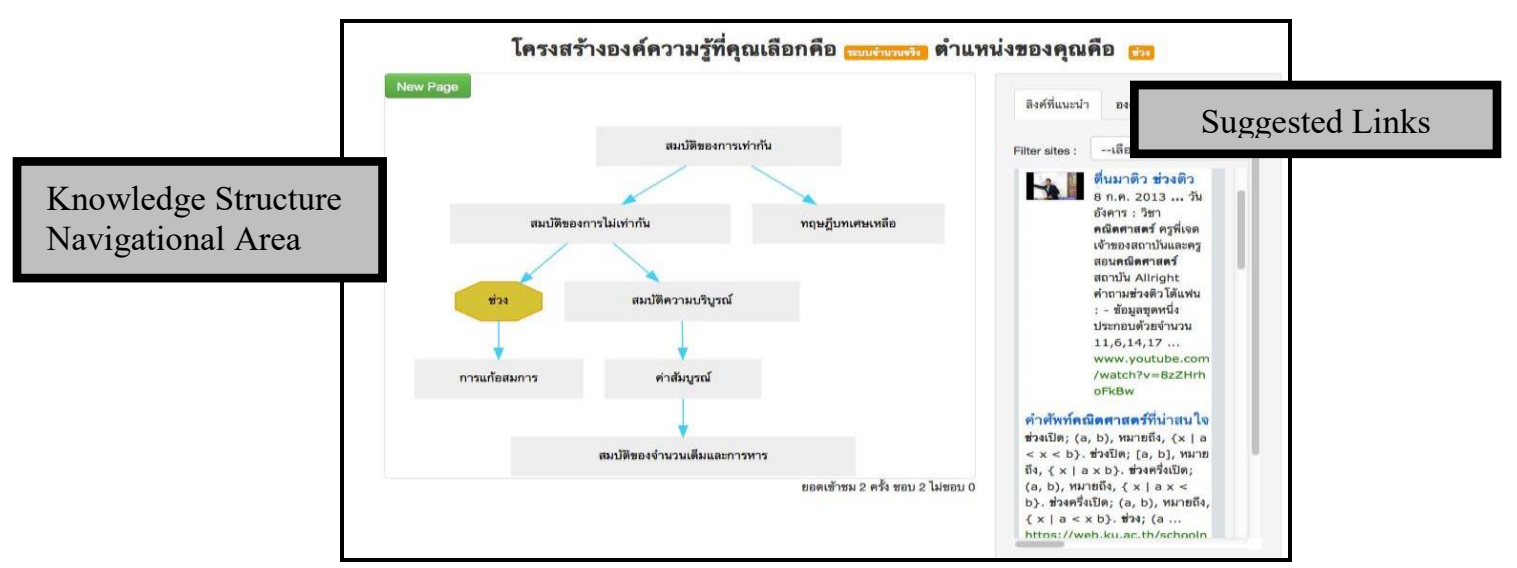

Fig. 5. Screenshot of the Prototype of Suggesting

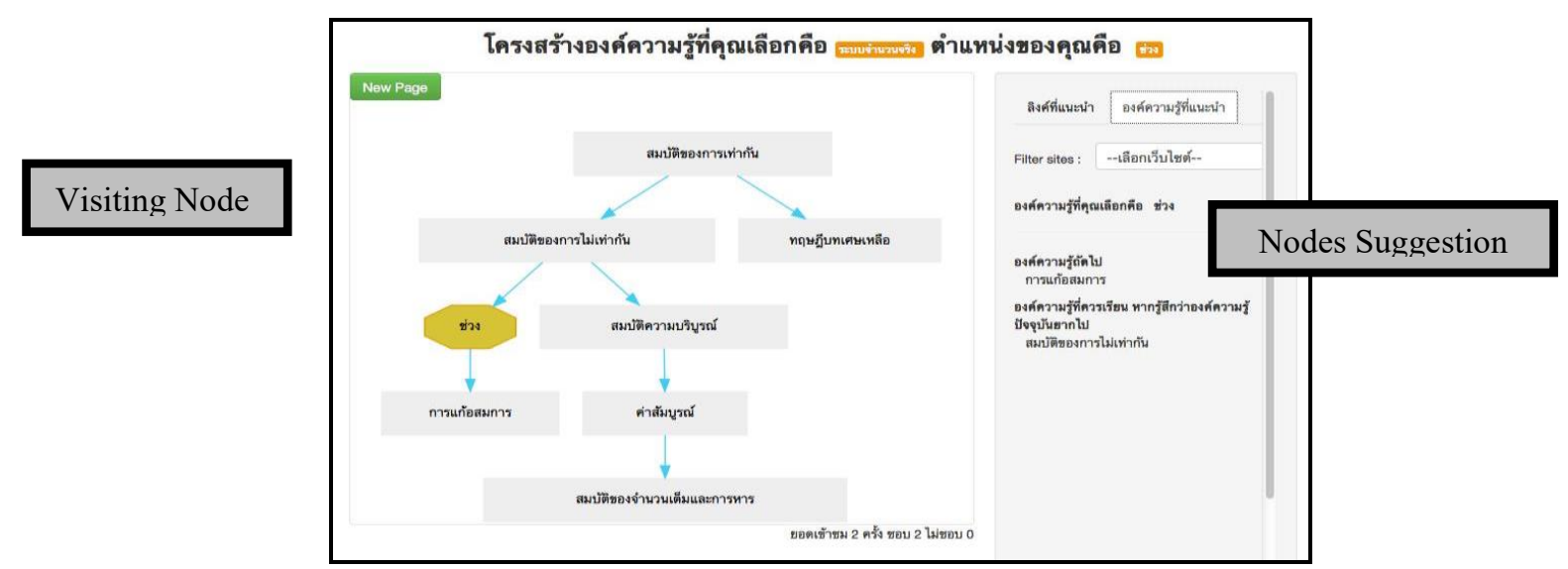

Fig. 6. Screenshot of the Prototype Gives Some Suggestions When Learner Click to Another Node 


\section{Experimental Design and Results}

A preliminary survey on teachers' experiences in using and designing knowledge structures was conducted, comprising a questionnaire and an interview. For the questionnaire study, participants were 173 school teachers from Songkhla province. The results showed that the teachers have used some tools for building such structures, such as a mind-mapping tool and the Cmap tool. Most of them (136 school teachers) recognized and used mind-maps during their teaching activities. Mind-maps are the tool for conceptualizing the user's thinking. Freely drawing is allowed. The survey shows that most structures they have built are mind maps. None of them represented or designed pedagogical structures with linkage among the knowledge components. They were interested in using a tool for building pedagogical knowledge structure (interested $=106$, highly interested $=61$ ) and were willing to use the structures and their applications as educational aids (interested $=101$, highly interested $=62$ ).

The interview study was conducted with 10 school teachers from the schools mentioned. Even though knowledge structures have been recommended for many years, school teachers still did not report using them in their teaching. However, the teachers felt that knowledge structures were useful and they could benefit education more. For example, such maps could help learners think about and understand the relationships among the information shown. On the teachers' sides, they thought that designing knowledge structure could be another way of sharing their tacit knowledge with other teachers.

Later, another experiment was conducted. This experiment is to determine experts' overall reaction to Kirkpatrick's level one 'reaction' against the tool developed in this research [28]. The analysis is to measure any significant difference in the mean rating for each dependent variable from the particular value ' 3 ' on the Likert scale. Overall participants are knowledge domain experts. All participants are high school mathematical teacher at Songkhla Province, Thailand. The estimated number of participants required was obtained using G*Power software [29]. The number of participants is 60 as shown in Table I.

The questionnaire is designed to ask experts to review and give a rating against the knowledge structure and system's features on a 5-point Likert scale, namely: 'Strongly Disagree', 'Disagree', 'Neither Agree Nor Disagree', 'Agree' and 'Strongly Agree'. The weighted ratings for each scale are 1, 2, 3, 4 and 5, respectively.

Table 1. Experimental Results

\begin{tabular}{|c|l|c|c|c|}
\hline No & \multicolumn{1}{|c|}{ Dependent Variables } & N & Mean & Sig. (2-tailed) \\
\hline 1 & Clarity of node appearance & 60 & 4.33 & $<0.07$ \\
\hline 2 & Clarity of the relationship between nodes & 60 & 4.25 & $<0.07$ \\
\hline 3 & Satisfaction on the relationship between nodes & 60 & 4.11 & $<0.07$ \\
\hline 4 & Tool is easy to use & 60 & 4.20 & $<0.07$ \\
\hline 5 & Overall user's satisfaction on tool & 60 & 4.38 & $<0.07$ \\
\hline 6 & Matching the materials with the structure nodes & 60 & 4.31 & $<0.07$ \\
\hline 7 & Suggestion for future use & 60 & 4.52 & $<0.07$ \\
\hline
\end{tabular}

The expected sample size for each experiment was 13, using the given values of: effect size f as 1 , alpha error probability as 0.05 , power as 0.95 , test-family as t-test and statistical test as Means-Difference from constant (one sample case). 
A one-sample t-test was used to analyze the data obtained for each variable in the experiment. For this experiment, the number of tests of significance $\mathrm{m}$ equals 8 . Bonferroni correction provides an $\alpha$ level of $0.05 / \mathrm{m}$ (where, $\mathrm{m}$ is number of dependent variables). Our criterion for significance was thus 0.0071 . For all dependent variables, the mean ratings for the tool were significantly higher than $3(\mathrm{p}<0.007)$ which is the middle, 'neutral', option.

From the experimental results, participants were in general significantly satisfied with the clarity of node and structure appearance. The experts were able to understand the appearance/design of the structures. They thought that the system provides a wide range of types of materials which are matched with the structure nodes.

\section{Conclusion and Future Works}

A knowledge-based system for suggesting study material links from the Web has been proposed in this research. The aim of the approach is to assist learners to achieve their desired knowledge. In addition, the system provides learners with the suggested knowledge can be identified based on the current and previously selected node. The method of constructing the knowledge structure is also proposed. This is to consider the nature and type of each knowledge in order to find the relationship among them. Experimental studies were conducted, which explored the expert reaction ratings against the approach and the results show that this research tool is overall acceptable for learners. However, there are some limitations such as, the system's dependent on search engine API. The coding function within such API tends to be changed regularly, which may affect the usability of the application. A future plan is to include a self-search engine with an application and improve the application's user interface and user experience. In addition, more knowledge domains will also be explored.

\section{References}

1. B. Yang and Y. Sun, "An exploration of link-based knowledge map in academic web space," Scientometrics, vol. 96, pp. 239-253, 2013/07/01 2013.

2. K.-K. Chu, C.-I. Lee, and R.-S. Tsai, "Ontology technology to assist learners' navigation in the concept map learning system," Expert Systems with Applications, vol. 38, pp. 11293-11299, 2011.

3. C.-C. Chiou, L.-T. Lee, and Y.-Q. Liu, "Effect of Novak Colorful Concept Map with Digital Teaching Materials on Student Academic Achievement," Procedia - Social and Behavioral Sciences, vol. 64, pp. 192-201, 2012.

4. L. Li-Yu, L. Yu-Shih, and C. Chih-Ping, "Constructing personal concept map automatically via Correlative TestItems Structure," in 11th International Conference on Information Technology Based Higher Education and Training (ITHET), Istanbul, Turkey, 2012, pp. 1-5.

5. A. Nitchot, L. Gilbert, and G. Wills, "Competence-based System for Recommending Study Materials from the Web: Design and Experiment," in World Conference on Educational Multimedia, Hypermedia and Telecommunications (ED-Media 2011), Lisbon, Portugal, 2011, pp. 2074-2083.

6. A. V. D'Antoni, G. P. Zipp, V. G. Olson, and T. F. Cahill, "Does the mind map learning strategy facilitate information retrieval and critical thinking in medical students?," BMC medical education, vol. 10, p. 61, 2010.

7. S. Saiyasombut and Siam Voices. (2012, 11/10/1012). Thai education failures - Part 3: PISA scores \& a challenge for the 21 st century. Available: http://asiancorrespondent.com/77060/thai-education-failures-part-3-pisa-scores-and-achallenge-for-the-21st-century/

8. S. Sirisoonthorn, "Why Thai students weak at Mathematics?," in bangkokbiznews.com, ed, 2012.

9. Parinya Plicharoensuk. (2012). Reasons why Thai students are weak in Mathematics. Available: http://www.kruparinya.com/index.asp?contentID $=10000004 \&$ title $=\% \mathrm{~A} 4 \% \mathrm{C} 7 \% \mathrm{D} 2 \% \mathrm{C} 1 \% \mathrm{CA} \% \mathrm{D} 3 \% \mathrm{~A} 4 \% \mathrm{D} 1 \% \mathrm{AD} \% \mathrm{E}$ 
1\%C5\%D0\%A4\%C7\%D2\%C1\%E0\%BB\%E7\%B9\%C1\%D2\%A2\%CD $\% \mathrm{~A} 7 \% \mathrm{BB} \% \mathrm{D} 1 \% \mathrm{AD} \% \mathrm{CB} \% \mathrm{D} 2 \&$ getarticle $=4$ $0 \&$ keyword $=\&$ catid $=1$

10. J. F. Sowa, Knowledge representation : logical, philosophical, and computational foundations: Pacific Grove, CA: Brooks/Cole, 2000.

11. J. D. Novak and A. J. Canas, "The Theory Underlying Concept Maps and How to Construct Them," Institute for Human and Machine Cognition, Pensacola, Florida, USA2006.

12. C.-H. Lee, G.-G. Lee, and Y. Leu, "Application of automatically constructed concept map of learning to conceptual diagnosis of e-learning," Expert Systems with Applications, vol. 36, pp. 1675-1684, 2009.

13. A. Nitchot, L. Gilbert, and G. B. Wills, "Competence Web-based System for Suggesting Study Materials Links: Approach \& Experimental Design," in World Conference on Educational Multimedia, Hypermedia and Telecommunications, Colorado, USA, 2012, pp. 1886-1895.

14. M. D. Kickmeier-Rust, D. Albert, and C. Steiner, "Lifelong Competence Development: On the Advantages of Formal Competence-Performance Modeling," in International Workshop on Learning Networks for Lifelong Competence Development, TENCompetence Conference, Sofia, Bulgaria, 2006.

15. D. Albert, C. Hockemeyer, B. Mayer, and C. M. Steiner, "Cognitive Structural Modelling of Skills for Technology-Enhanced Learning," in 7th IEEE International Conference on Advanced Learning Technologies (ICALT 2007) Beijing, China, 2007, pp. 322-324.

16. M. D. Engelhart, E. J. Furst, W. H. Hill, and D. R. Krathwohl, Taxonomy of Educational Objectives, Handbook I : The cognitive domain. New York: David McKay, 1956.

17. R.-S. Shaw, "A study of learning performance of e-learning materials design with knowledge maps," Computers \& Education, vol. 54, pp. 253-264, 2010.

18. M. A. Ruiz-Primo, S. E. Schultz, M. Li, and R. J. Shavelson, "Comparison of the reliability and validity of scores from two concept-mapping techniques," Journal of Research in Science Teaching, vol. 38, pp. 260-278, 2001.

19. Z. Yun, Z. Chenghong, F. Tianjun, and L. Songran, "An Approach for Constructing Knowledge Map Embedded in the Social Relationship Network," in International Conference on E-Business and E-Government (ICEE), Guangzhou, China, 2010, pp. 1750-1754.

20. M. Carvalho, R. Hewett, and A. J. Cañas, "Enhancing Web Searches from Concept Map-based Knowledge Models," in 5th Multi-Conference on Systems, Cybernetics and Informatics (SCI 2001), Orlando, Florida, USA, 2001. 21. S.-H. Liu and G.-G. Lee, "Using a concept map knowledge management system to enhance the learning of biology," Computers \& Education, vol. 68, pp. 105-116, 2013.

22. C.-C. Lee, B.-J. Liu, and C.-C. Chang, "Integrated Learning Courses and Library Resources with Personal Knowledge Map," Advanced Materials Research, vol. 403-408, pp. 5146-5149, 2011.

23. L. Gilbert and V. Gale, Principles of E-learning Systems Engineering. Oxford, UK: Chandos Publishing, 2008.

24. A. Nitchot, L. Gilbert, and G. B. Wills, "Constructing a Competence Structure in Recommending Study Materials Links," International Journal of Advances in Computer and its Applications (IJCSIA), vol. 2, pp. 295-299, 2013.

25. M. D. Merrill, Instructional Design Theory. New Jersey: Educational Technology Publication, 1994.

26. J. Ellson, E. R. Gansner, E. Koutsofios, S. C. North, and G. Woodhull, "Graphviz and Dynagraph -Static and Dynamic Graph Drawing Tools," in Graph Drawing Software, M. Junger and P. Mutzel, Eds., ed Berlin/Heidelberg, 2004, pp. 127-148.

27. L. Nachmanson. (2015, 22/7/2015). Microsoft Automatic Graph Layout. Available: http://research.microsoft.com/en-us/projects/msagl/

28. D. L. Kirkpatrick, Implementing the four levels : a practical guide for effective evaluation of training programs 1ed.: San Francisco : Berrett-Koehler Publishers, 2007.

29. A. Buchner, F. Faul, and E. Erdfelder. (2010, 09/09/2010). G*Power Official Page. Available: http://www.psycho.uni-duesseldorf.de/aap/projects/gpower/ 